\title{
Search for electroweak SUSY production at CMS
}

\author{
Hamed Bakhshiansohi* ${ }^{* \dagger}$ \\ Institute for research in fundamental sciences (IPM), Tehran, Iran \\ E-mail: Hamed.Bakhshian@cern.ch
}

\begin{abstract}
A review of recent results from CMS on searches for the electroweak production of supersymmetry particles are presented. All the possible production scenarios including $\chi^{ \pm} \chi^{\mp}, \chi^{0} \chi^{ \pm}, \chi^{0} \chi^{0}$ and $\tilde{l} \tilde{l}$ are investigated. The full 2012 dataset which comprises $19.6 \mathrm{fb}^{-1}$ of proton-proton collisions at $\sqrt{s}=8 \mathrm{TeV}$ is used. In all studied decay modes, the observed yield agrees well with the expectation of the Standard Model (SM) and limits are set on the rates of the direct production of charginos, neutralinos and sleptons.
\end{abstract}

XXII. International Workshop on Deep-Inelastic Scattering and Related Subjects, 28 April - 2 May 2014

Warsaw, Poland

\footnotetext{
*Speaker.

$\dagger$ on behalf of the CMS Collaboration
} 


\section{Introduction}

Most of the searches for Super Symmetry (SUSY) [1] at the Compact Muon Solenoid (CMS) [2] have focused on the production of gluinos and squarks productions, the SUSY partners of the gluon and quarks, via strong interactions $[3,4,5]$. Observing no evidence of new physics, the stronglyinteracting SUSY particles up to a mass scale of $\sim 1 \mathrm{TeV}$ are excluded. If squarks and gluinos are heavy, direct electroweak production of charginos and neutralinos, the SUSY partners of the $W^{ \pm}$, $Z$, and Higgs bosons, may be dominated at the LHC. Although the electroweak SUSY production may suffer from the very low rate, the clean leptonic final state and the QCD-free selection makes this study possible.

\section{2. $\tilde{\chi}^{ \pm} \tilde{\chi}_{2}^{0}$ searches}

The simultaneous production of a chargino and a neutralino has the highest rate among electroweak SUSY productions. The mass of the $\tilde{\chi}^{ \pm}$and $\tilde{\chi}_{2}^{0}$ are assumed to be equal for this study. Different decay scenarios for this study are considered.

\subsection{Decay to sleptons}

When the slepton mass is between the $\tilde{\chi}^{ \pm}$and LSP $\left(\tilde{\chi}_{1}^{0}\right)$ masses, the parent SUSY particle decays to leptons via sleptons. Three possible decay chains are shown in Fig. 1. The final state of such decay would be three leptons together missing transverse energy $\left(\vec{E}_{\mathrm{T}}^{\text {miss }}\right)$.

For selecting signal events, all events with 3 leptons and $\vec{E}_{\mathrm{T}}^{\text {miss }}>50(\mathrm{GeV})$ are selected. To suppress $t \bar{t}$ events, events with at least one b-tagged jet are discarded. The $W Z$ contribution is estimated using simulated samples where a data driven fake rate method is exploited to estimate the $t \bar{t}$ background. Events are classified based on lepton flavors, the invariant mass of the leptons and $\vec{E}_{\mathrm{T}}^{\text {miss }}[6]$.

For the compressed spectrum, when the slepton mass is close to the LSP mass, the produced lepton could be so soft that escapes detection. To cover this part of the phase space, a search for events with two same-sign leptons plus missing energy is also performed [6].

Results are used to set exclusion limits on the SUSY particle masses (Fig. 3).
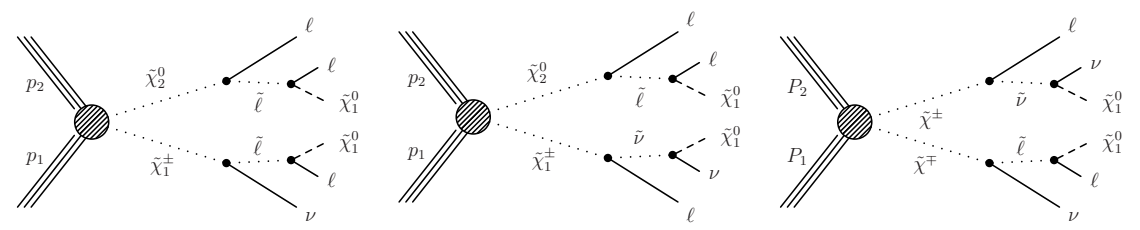

Figure 1: Possible decay modes of chargino and neutralino when they are heavier than sleptons.

\subsection{Decay to $W$ and $Z$ bosons}

The SUSY parent particles decay to $Z$ and $W$ bosons if it is forbidden to decay to sleptons and if the neutralino is bino-like (Fig. 2 (Left)). The leptonic decay of the $Z$ boson together with 
leptonic and hadronic decays of the $W$ boson are taken into account for this study. For the leptonic decay of the $W$ boson, the 3 lepton selection which is described in the previous section is used.

To select $W Z$ events where the $W$ boson decays hadronically, events with two leptons, compatible with a $Z$ boson and 2 jets compatible with a $W$ boson are selected. A cut on $\vec{E}_{\mathrm{T}}^{\text {miss }}$ is applied and events with $\vec{E}_{\mathrm{T}}^{\text {miss }}>80 \mathrm{GeV}$ are selected. The $t \bar{t}$ contamination is suppressed via the b-Jet veto. The $Z+$ Jets and $t \bar{t}$ backgrounds are estimated using data driven methods [6].

Results are used to set exclusion limits on the SUSY particle masses (Fig. 3).

\subsection{Decay to $W$ and Higgs bosons}

Discovering the SM Higgs boson motivates the search for a Higgsino-like neutralino (Fig. 2 (Right)). The leptonic decay of the $W$ boson along with different Higgs decays are studied [7]. Fig. 3 (Left) shows the cross section of the $W H$ production multiplied by the branching fraction for different decay modes of the $W$ and the Higgs bosons vs. the mass of the chargino and neutralino.

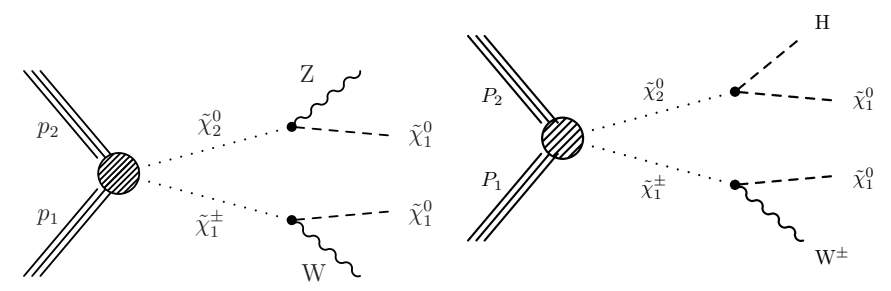

Figure 2: When sleptons are heavier than chargino and neutralino, they decay to SM bosons.

For the Higgs bosons decaying to $b \bar{b}$, events with 1 lepton and exactly two b-tagged jets are selected. Moderate cuts on $\vec{E}_{\mathrm{T}}^{\text {miss }}$ and the transverse mass of the lepton are applied. All the backgrounds are estimated using simulation. A peak is searched for in the invariant mass of the $b \bar{b}$.

To search for $H \rightarrow W(l v) W(j j)$, events with two same-sign leptons and two jets are selected. For the backgrounds from fake leptons, a data driven method is employed. All backgrounds with 2 prompt same-sign leptons are taken from MC. In the invariant mass of the lepton and two jets a search is performed to find the Higgs peak.

As no evidence of new physics is observed, the results are used to set exclusion limits on the mass of the chargino and neutralino (Fig 3 (Right))

\section{3. $\tilde{\chi}^{ \pm} \tilde{\chi}^{\mp}$ and $\tilde{l}^{ \pm} \tilde{l}^{\mp}$ searches}

As it is presented in Fig. 4, the final state for $\tilde{\chi}^{ \pm} \tilde{\chi}^{\mp}$ and $\tilde{l}^{ \pm} \tilde{l}^{\mp}$ processes are two opposite sign leptons with $\vec{E}_{\mathrm{T}}^{\text {miss }}$. While the leptons from $\tilde{l}^{ \pm} \tilde{l}^{\mp}$ have the same flavor, $\tilde{\chi}^{ \pm} \tilde{\chi}^{\mp}$ can produce oppositeflavor leptons too. So events with two high $p_{T}$ opposite-sign leptons (e or $\mu$ ) and a moderate $\vec{E}_{\mathrm{T}}^{\text {miss }}$ are selected. Events under the $Z$ boson mass peak are discarded. The b-Jet veto is also applied to suppress $t \bar{t}$ events [6].

As it is shown in Fig. 5, the distribution of $M_{C T \perp}$ variable [8] can be used to separate the $W W$ and other backgrounds from signal. Fitting over the template distribution of this variable for different backgrounds shows a very good consistency between data and simulation and so the yields are used to set exclusion limits on the mass of $\tilde{\chi}^{ \pm}$and $\tilde{l}$. The results are shown in Fig. 6. 

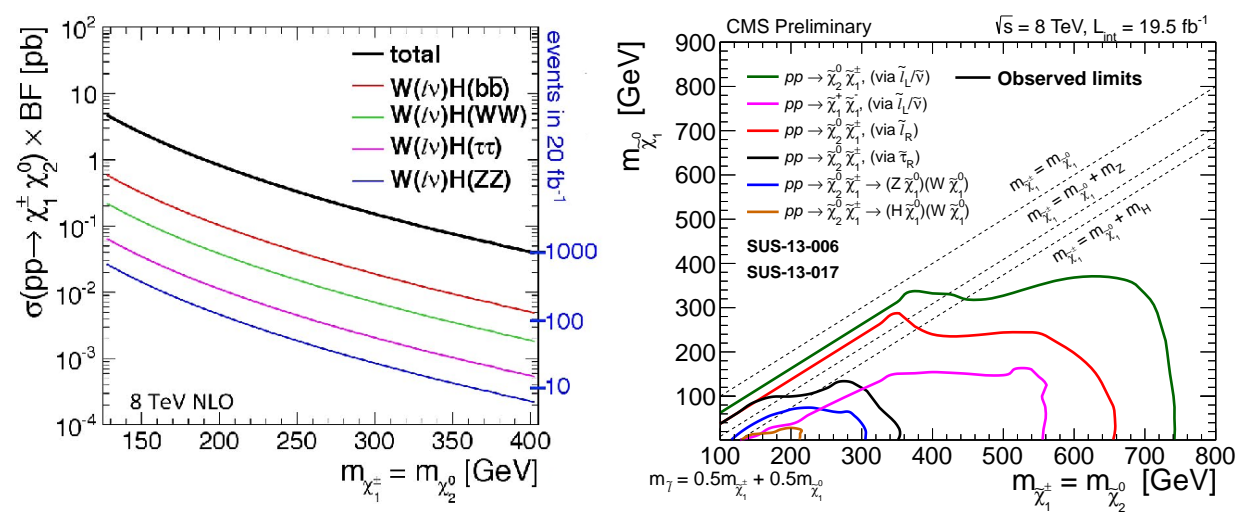

Figure 3: Cross section of $W H$ via SUSY electroweak production vs. the $\tilde{\chi}^{ \pm}$mass for different branching fractions (Left) and the exclusion limits on the mass of the chargino and neutralino in $\tilde{\chi}^{ \pm} \tilde{\chi}_{2}^{0}$ searches by the CMS collaboration (Right).
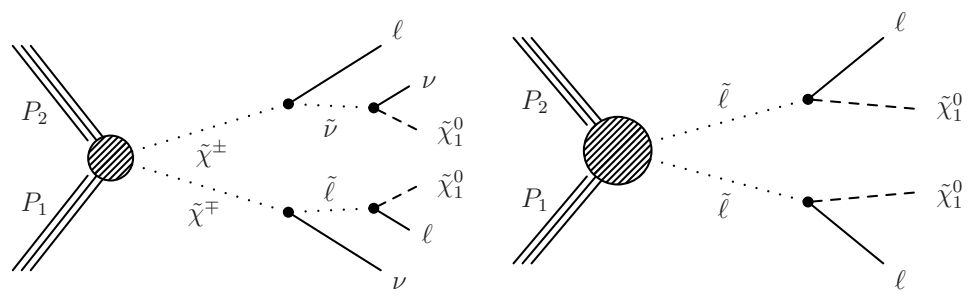

Figure 4: $\tilde{\chi}^{ \pm} \tilde{\chi}^{\mp}$ and $\tilde{l}^{ \pm} \tilde{l}^{\mp}$ decay diagrams.

\section{Searching for $\tilde{\chi}^{0} \tilde{\chi}^{0}$ production}

When $\tilde{\chi}^{0}$ and $\tilde{\chi}^{ \pm}$are both wino-like, the production of $\tilde{\chi}^{0} \tilde{\chi}^{0}$ is very suppressed. In gaugemediated-SUSY-breaking (GMSB) models $[9,10,11]$ the production rate of this process is enhanced. LSP in this model is an almost massless gravitino. In the scenario with $Z$-enriched Higgsino, $\tilde{\chi}^{0}$ decays mostly to LSP and a $Z$ boson and so the final state would be $2 Z$ bosons and $\vec{E}_{\mathrm{T}}^{\text {miss }}$ (Fig. 7 (Left)). For the case of Higgsino-like $\tilde{\chi}^{0}$, two produced $\tilde{\chi}^{0}$ s decay to two Higgs bosons and $\vec{E}_{\mathrm{T}}^{\text {miss }}$ according to Fig. 7 (Right).

\section{1 $\tilde{\chi}^{0} \tilde{\chi}^{0} \rightarrow Z Z+\vec{E}_{\mathrm{T}}^{\mathrm{miss}}$}

Events with 4 leptons (e, $\mu$ and up to one hadronically decaying $\tau$ lepton) in the final sate are selected [6]. At least one ee or $\mu \mu$ pair consistent with the $Z$ boson mass is requested. Events are categorized according to the number of opposite-sign and same-flavor pairs, number of hadronic $\tau$ 's and $\vec{E}_{\mathrm{T}}^{\text {miss }}$. As no excess over expectation is observed, this results together with the $2 l+2 j e t s$ selection are used to exclude the Higgsino mass in the GMSB models (Fig. 8 (Left)).

$4.2 \tilde{\chi}^{0} \tilde{\chi}^{0} \rightarrow H H+\vec{E}_{\mathrm{T}}^{\text {miss }}$

As the Higgs boson decays in $60 \%$ of cases into two b-quarks, only the $H \rightarrow b \bar{b}$ decay is 


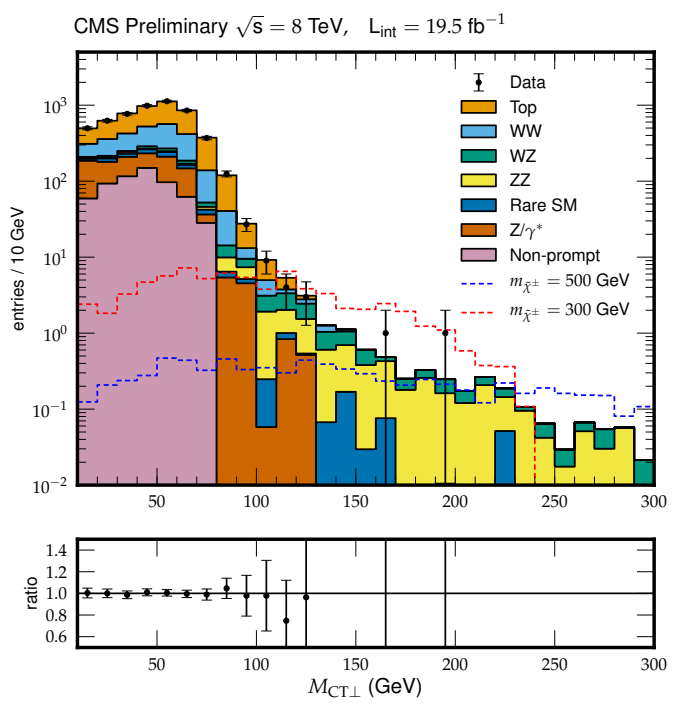

Figure 5: The distribution of $M_{C T \perp}$ variable for the selected events and different backgrounds and two sample signal points.
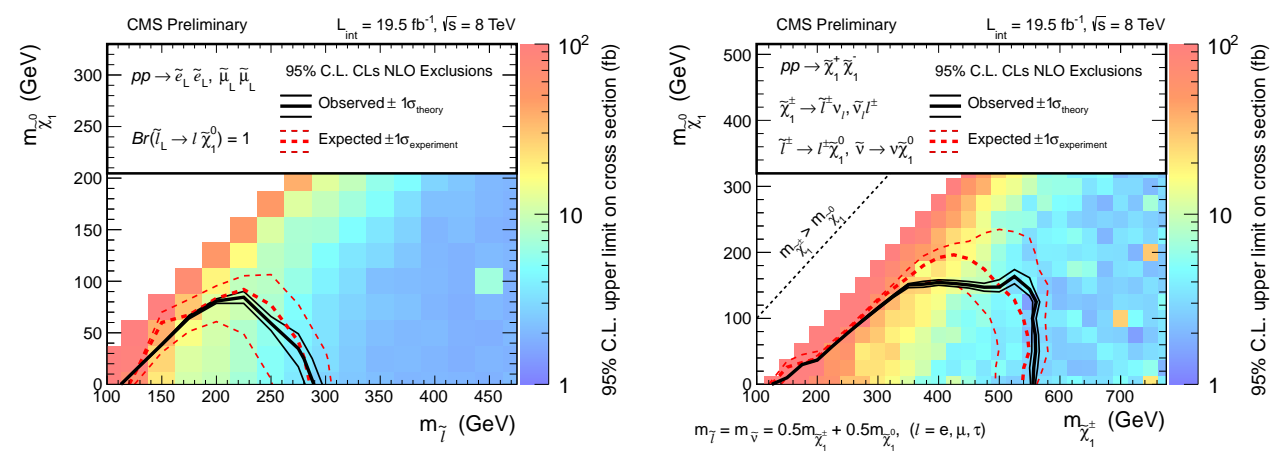

Figure 6: The exclusion plots for the $\tilde{\chi}^{ \pm} \tilde{\chi}^{\mp}$ (Left) and $\tilde{l}^{ \pm} \tilde{l}^{\mp}$ (Right).

considered for this search [12]. Events with 4 or 5 jets in the final state are selected among which at least 3 jets have to be tagged as b-quark jet. To reconstruct the Higgs bosons, the combination of jets minimizing $\left|\Delta m_{j j}\right| \equiv\left|m_{j j, 1}-m_{j j, 2}\right|$ is selected. To select events with $m_{j j, 1}$ and $m_{j j, 2}$ consistent with the SM Higgs mass, two cuts are applied : $100<\left\langle m_{j j}\right\rangle<140 \mathrm{GeV}$ and $\left|\Delta m_{j j}\right|<20 \mathrm{GeV}$. To estimate the backgrounds which is mostly $t \bar{t},\left\langle m_{j j}\right\rangle$ and $\left|\Delta m_{j j}\right|$ variables are used to define the signal and control regions.

The yields in different $\vec{E}_{\mathrm{T}}^{\text {miss }}$ ranges are compared to the background expectation and no sign of new physics is observed. However, because of a slight excess of data events compared to the background expectation we are unable to exclude the signal hypothesis for any value of Higgsino mass. Figure 8 (Right) shows the results in terms of the signal model cross section. 


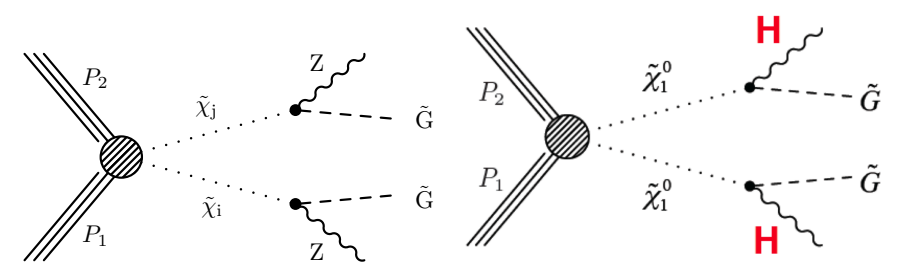

Figure 7: $\tilde{\chi}^{0} \tilde{\chi}^{0}$ possible decay modes.
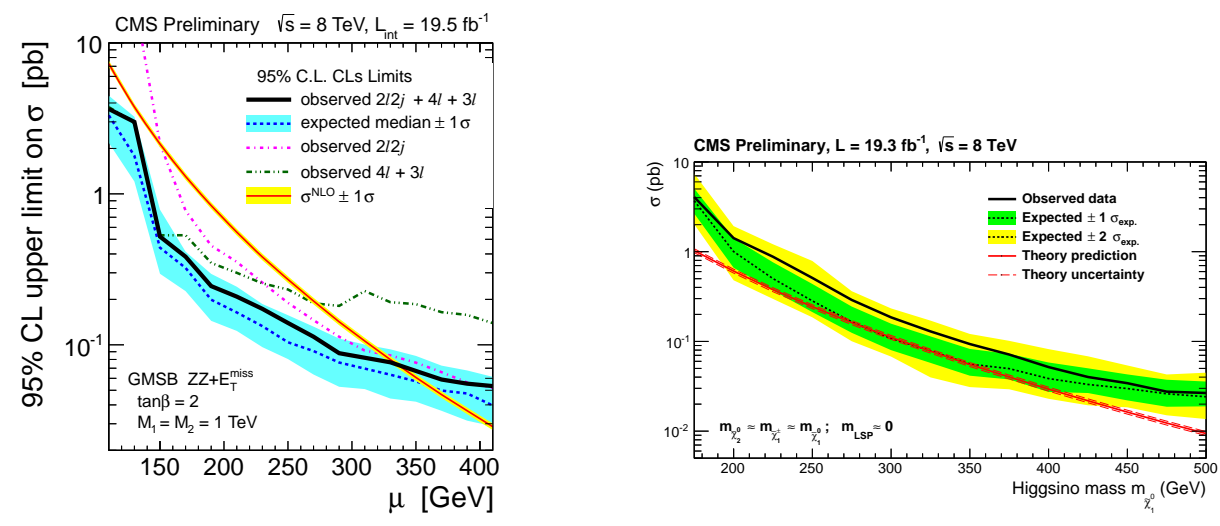

Figure 8: Expected and observed upper limits on the cross section of the $\tilde{\chi}^{0} \tilde{\chi}^{0}$ production for $Z Z+\vec{E}_{\mathrm{T}}^{\text {miss }}$ (Left) and $H H+\vec{E}_{\mathrm{T}}^{\text {miss }}$ (Right) studies.

\section{References}

[1] Yu.A. Golfand and E.P. Likhtman. "Extension of the algebra of Poincare group generators and violation of p invariance". In: JETP Lett. 13 (1971), p. 323.

[2] CMS Collaboration. "The CMS experiment at the CERN LHC". In: Journal of Instrumentation 3.08 (2008), S08004. URL: http: / / stacks.iop.org/1748-0221/3/i=08/ $\mathrm{a}=\mathrm{S} 08004$.

[3] CMS Collaboration. "Search for new physics in events with same-sign dileptons and $b$ tagged jets in $p p$ collisions at $\sqrt{s}=7 \mathrm{TeV}$ ". In: JHEP 08 (2012), p. 110. DOI: 10.1007 / JHEP 08 (2012) 110. arXiv:1205.3933 [hep-ex] .

[4] CMS Collaboration. "Search for new physics in events with opposite-sign leptons, jets, and missing transverse energy in $p p$ collisions at $\sqrt{s}=7 \mathrm{TeV}$ ". In: Phys. Lett. B 718 (2013), pp. 815-840. DoI: $10.1016 /$ j . physletb. 2012 . 11 .036. arXiv:1206.3949 [hep-ex].

[5] CMS Collaboration. "Search for new physics with same-sign isolated dilepton events with jets and missing transverse energy". In: Phys. Rev. Lett. 109 (2012), p. 071803. DOI: 10 . 1103/PhysRevLett.109.071803. arXiv:1205.6615 [hep-ex]. 
[6] CMS Collaboration. Search for electroweak production of charginos, neutralinos, and sleptons using leptonic final states in pp collisions at $8 \mathrm{TeV}$. Tech. rep. CMS-PAS-SUS-13-006. Geneva: CERN, 2013.

[7] CMS Collaboration. Search for electroweak production of charginos and neutralinos in final states with a Higgs boson in pp collisions at 8 TeV. Tech. rep. CMS-PAS-SUS-13-017. Geneva: CERN, 2013.

[8] Konstantin T. Matchev and Myeonghun Park. "A General method for determining the masses of semi-invisibly decaying particles at hadron colliders". In: Phys. Rev. Lett. 107 (2011), p. 061801. DOI: 10.1103/PhysRevLett.107.061801.arXiv:0910.1584 [hep-ph] .

[9] Konstantin T. Matchev and Scott D. Thomas. "Higgs and $Z$ boson signatures of supersymmetry". In: Phys. Rev. D 62 (2000), p. 077702. DOI: 10.1103 / PhysRevD . 62.077702.

[10] Patrick Meade, Matthew Reece, and David Shih. "Prompt decays of general neutralino NLSPs at the Tevatron". In: JHEP 05 (2010), p. 105. DOI: 10.1007 / JHEP 05 (2010) 105.

[11] Joshua T. Ruderman and David Shih. "General neutralino NLSPs at the early LHC”. 2011.

[12] CMS Collaboration. Search for electroweak production of higgsinos in channels with two Higgs bosons decaying to b quarks in pp collisions at $8 \mathrm{TeV}$. Tech. rep. CMS-PAS-SUS-13022. Geneva: CERN, 2014. 